\title{
Global Stability Analysis of the Original Cellular Model of Hepatitis C Virus Infection Under Therapy
}

\author{
Alexis Nangue \\ Department of Mathematics, Higher Teachers' Training College, University of Maroua, Maroua, Cameroon \\ Email address: \\ alexnanga02@yahoo.fr \\ To cite this article: \\ Alexis Nangue. Global Stability Analysis of the Original Cellular Model of Hepatitis C Virus Infection Under Therapy. American Journal of \\ Mathematical and Computer Modelling. Vol. 4, No. 3, 2019, pp. 58-65. doi: 10.11648/j.ajmcm.20190403.12
}

Received: June 12, 2019; Accepted: July 27, 2019; Published: August 29, 2019

\begin{abstract}
In this work, we investigate the hepatitis $\mathrm{C}$ virus infection under treatment. We first derive a nonlinear ordinary differential equation model for the studied biological phenomenon. The obtained initial value problem is completely analysed. To begin with the analysis of the model, we use the standard theory of ordinary differential equations to prove existence, uniqueness and boundedness of the solution. Morever, the basic reproduction number $R_{0}$ determining the extinction or the persistence of the $\mathrm{HCV}$ infection is computed and used to express the equilibrium points. Also the global asymptotic stability of the $\mathrm{HCV}$-uninfected equilibrium point and the HCV-infected equilibrium point of the model are derived by means of appropriate Lyapunov functions. Finally numerical simulations are carried out to confirm theoretical results obtained at HCV-unfected equilibrium.
\end{abstract}

Keywords: HCV Cellular Model, Differential System, Therapy, Local and Global Solution, Invariant Set, Stability

\section{Introduction}

According to [15] recent estimates, more than 185 million people around the world have been infected with the hepatitis $\mathrm{C}$ virus (HCV), of whom 350000 die each year. One third of those who become chronically infected are predicted to develop liver cirrhosis or hepatocellular carcinoma. Despite the high prevalence of disease, most people infected with the virus are unaware of their infection. For many who have been diagnosed, treatment remains unavailable. Treatment is successful in the majority of persons treated, and treatment success rates among patients treated in low- and middle-income countries are similar to those in high-income countries Hepatitis C virus (HCV) infects liver cells (hepatocytes). Approximately 200 million people worldwide are persistently infected with the $\mathrm{HCV}$ and are at risk of developing chronic liver disease, cirrhosis and hepatocellular carcinoma. HCV infection therefore represents a significants global public health problem. HCV established chronic hepatitis in $60 \%-80 \%$ of infected adults [12].

In literature, several mathematical models have been introduced for understanding HCV temporal dynamics $[4,9,10]$.

In this article, we consider the basic extracellular model with therapy presented by Neumann et al. in [9]. Given the recent surge in the development of new direct acting antivirals agents for HCV therapy, mathematical modelling of viral kinetics under treatment continues to play an instrumental role in improving our knowledge and understanding of virus pathogenesis and in guiding drug development $[2,7,11]$.

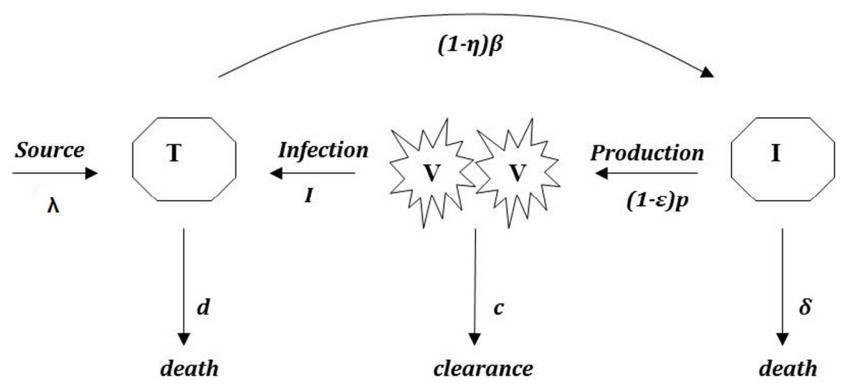

Figure 1. Schematic representation of the original viral kinetic model of $\mathrm{HCV}$ infection under treatment. $T$ represents the target uninfected cells, I is the infected cells and $V$ represents the free virus.

To proceed, we assume that the uninfected target cells are produced at a rate $\lambda$, die at constant rate $d$ per cell. On the other hand, the target cells are infected with de novo infection rate constant of $\beta$ and the infected cells die at a constant rate of $\delta$ per cell. The hepatitis $\mathrm{C}$ virions are produced inside the infected cells at an average rate $p$ per infected cell and have a constant 
clearance rate $c$ per virion. Thereby, viral persistence will occur when rate of viral production $(p)$, de novo infection $(\beta)$, and production of target cells $(\lambda)$ exceeds the clearance rate $(c)$, death rate of infected cells $(\delta)$ and target cells death rate $(d)$. In addition, the therapeutic effect of IFN treatment in this model involved blocking virions production and reducing new infections which, are described in fractions $(1-\varepsilon)$ and $(1-\eta)$, respectively. $(0 \leq \varepsilon \leq 1,0 \leq \eta \leq 1)$.

According to $[3,9]$, the above assumptions lead to the following differential equations:

$$
\begin{gathered}
\frac{d T}{d t}=\lambda-d T-(1-\eta) \beta V T ; \\
\frac{d I}{d t}=(1-\eta) \beta V T-\delta I ; \\
\frac{d V}{d t}=(1-\varepsilon) p I-c V ;
\end{gathered}
$$

where the equations relate the dynamics relationship between, $\mathrm{T}$ as the uninfected target cells (hepatocytes), I as the infected cells and $\mathrm{V}$ as the viral load (amount of viruses present in the blood). In this article, model system (1) is taken as the original model used to analyse the HCV dynamics.

The initial conditions associated to system (1) are given by:

$$
T\left(t_{0}\right)=T_{0}, I\left(t_{0}\right)=I_{0}, V\left(t_{0}\right)=V_{0}, t_{0} \in[0,+\infty] .
$$

This paper is organized as follows: The global properties of the solutions to the mathematical model is carried out in Section 2. The stability of the disease non-infected steady state, and the infected steady state is analysed in section 3 and finally in section 4 , some numerical simulations are carried out.

\section{Properties of Solutions of the Initial Value Problem (1)-(4)}

\subsection{Existence of Local Solutions}

The first step in examining model (1), (2), (3) is to prove that local solution of the initial-value problem does, in fact, exist, and that this solution is unique.

Theorem 2.1 Let $T_{0}, I_{0}, V_{0} \in R$ be given. There exists $t_{1}>t_{0}>0$ and continuously differentiable functions $T, I$, $V:\left[0, t_{0}\right) \rightarrow R$ such that the ordered triple $(T, I, V)$ satisfies (1), (2), (3) and $\left(T\left(t_{0}\right), I\left(t_{0}\right), V\left(t_{0}\right)\right)=\left(T_{0}, I_{0}, V_{0}\right)$.

To prove the result, we use the classical Cauchy-Lipschitz theorem. Since the first order system of ordinary differential equations (1), (2), (3) is autonomous, it suffices to show that the function $H: R^{3} \rightarrow R^{3}$ defined by:

$$
\begin{gathered}
H(x)=\left(\begin{array}{l}
h_{1}\left(x_{1}, x_{2}, x_{3}\right) \\
h_{2}\left(x_{1}, x_{2}, x_{3}\right) \\
h_{3}\left(x_{1}, x_{2}, x_{3}\right)
\end{array}\right)=\left(\begin{array}{l}
\lambda-d x_{1}-(1-\eta) \beta x_{3} x_{1} \\
(1-\eta) \beta x_{3} x_{1}-\delta x_{2} \\
(1-\varepsilon) p x_{2}-c x_{3}
\end{array}\right) \text { (5) } \begin{array}{l}
\text { non-negative. Using this and the } \\
\text { have: }
\end{array} \\
\left\{\begin{array} { l } 
{ \frac { d I } { d t } = - I \delta + ( 1 - \eta ) \beta V T } \\
{ \frac { d V } { d t } = - V c + ( 1 - \varepsilon ) p I }
\end{array} \Rightarrow \left\{\begin{array} { l } 
{ \frac { d I } { d t } \geq - I \delta } \\
{ \frac { d V } { d t } \geq - V c }
\end{array} \Rightarrow \left\{\begin{array}{l}
\frac{d}{d t}(\ln I(t)) \geq-\delta \\
\frac{d}{d t}(\ln V(t)) \geq-c,
\end{array}\right.\right.\right.
\end{gathered}
$$

is locally Lipschitz in its $x$ argument. In fact, it is enough to notice that the jacobian matrix

$$
\nabla H(x)=\left[\begin{array}{lll}
-d-(1-\eta) \beta x_{3} & 0 & -(1-\eta) \beta x_{1} \\
(1-\eta) \beta x_{3} & -\delta & (1-\eta) \beta x_{1} \\
(1-\varepsilon) p & 0 & -c
\end{array}\right]
$$

is locally bounded for every $x \in R^{3}$. Hence, $\mathrm{H}$ has a continuous, bounded derivative on any compact subset of $R^{3}$ and so $\mathrm{H}$ is locally Lipschitz in $x$; In addition $\mathrm{H}$ is continuous. By Cauchy-Lipschitz theorem, there exists a unique solution, $x(t)$, to the ordinary differential equation

$$
x^{\prime}(t)=H(x(t))
$$

with initial value $x\left(t_{0}\right)=x_{0}$ on $\left[t_{0}, t_{1}\right]$ for some time $t_{1}>t_{0} \geq 0$.

Remark 1 The model (1), (2), (3) can be rewrite in the form

$$
S^{\prime}(t)=H(S(t))
$$

where $S(t)=(T(t), I(t), V(t))^{t}$ and $\mathrm{H}$ is defined by (5).

Remark 2 Since $\mathrm{H}$ is a continuously differentiable function, we deduce a unique maximal solution of initial value problem (1)-(4). In addition, F, being indefinitely continuously differentiable, we can also deduce that this solution is also only if indefinitely continuously differentiable.

Additionally, we may show that for positive initial data, solutions of ininitial value problem (1)- (4) remain positive as long as they exist.

\subsection{Positivity}

Theorem 2.2 Let $(T, I, V)$ be a solution of the Cauchy problem (1)-(4) on an interval $\left[t_{0}, t_{1}\right]$. Assume the initial data of problem (1)- (4) satisfy $T_{0}>0, I_{0}>0$, and $V_{0}>0$ then $T(t), T(t)$ and $V(t)$ remain positive for all $t \in\left[t_{0}, t_{1}\right]$.

Proof. Call the variables $x_{i}$. If there is an index $i$ and a time $t$ for which $x_{i}(t)=0$, let $t_{*}$ be the infimum of all such $t$ for any $\mathrm{i}$. Then the restriction of the solution to the interval $\left[t_{0} ; t_{*}\right]$ is positive and $x_{i}\left(t_{*}\right)=0$ for a certain value of $i$. The equation for $x_{i}$ in the system (1), (2), (3) can be written in the form:

$$
\frac{d x_{i}(t)}{d t}=-x_{i} f(x)+g(x)
$$

where $g(x)$ is non-negative. As a consequence $\frac{d x_{i}(t)}{d t} \geq$ $-x_{i} f(x)$ and $x_{i}(t)>0, \forall t \in\left[t_{0}, t_{*}\right]$. In fact:

Recall that all constants in the system (1), (2), (3) are non-negative. Using this and the solutions on $\left[t_{0}, t_{*}\right]$, we 
which yields:

$$
\begin{gathered}
I(t) \geq I_{0} e^{-\delta\left(t-t_{0}\right)}, \\
V(t) \geq V_{0} e^{-c\left(t-t_{0}\right)} \text { for all } t \in\left[t_{0}, t^{*}\right] .
\end{gathered}
$$$$
\frac{d T}{d t}=\lambda-d T-\operatorname{red}(1-\eta) \beta V T \leq \lambda .
$$

Solving for T yields

Similarly, in one hand we have:

$$
T(t) \leq T_{0}+\lambda\left(t-t_{0}\right) \leq A_{1}\left(1+\left(t-t_{0}\right)\right)
$$

where $A_{1} \geq \max \left\{T_{0}, \lambda\right\}$. In other hand we have:

$$
\frac{d}{d t}(I+V)=-I \delta+(1-\eta) \beta V T-V c+(1-\varepsilon) p I \leq \beta V T+p I .
$$

Recall that we have a bound on $\mathrm{T}$, so

$$
\frac{d}{d t}(I+V) \leq A_{1}\left(1+\left(t-t_{0}\right)\right) \beta T+p I \leq A_{2}\left(1+\left(t-t_{0}\right)\right)(T+I)
$$

where $A_{2} \geq \max \left\{A_{1} \beta, p\right\}$. Solving the differential inequation yields:

$$
I(t)+V(t) \leq A_{3} H_{1}(t), \forall t \in\left[t_{0}, t^{*}\right]
$$

where $A_{3}>0$ depends upon $A_{2}, I_{0}$ and $V_{0}$ only, and $H_{1}(t)=e^{\int_{t_{0}}^{t}\left(1+\left(t-t_{0}\right)\right) d \tau}>0, t \in\left[t_{0}, \mathrm{t}_{*}\right]$. Using the fact that $I(t)$ and $V(t)$ are positive, (6) yields:

$$
A_{3} H_{1}(t) \geq I(t)+V(t) \geq V(t), A_{3} H_{1}(t) \geq I(t)+V(t) \geq I(t) .
$$

With these bounds in place, we can now examine $T(t)$ and bound it from below using:

$$
\begin{gathered}
\frac{d T}{d t}=\lambda-d T-(1-\eta) \beta V T \\
\geq-d T-(1-\eta) \beta V T \\
\geq-d T-(1-\eta) \beta A_{3} H_{1}(t) T \\
\frac{d T}{d t} \geq-A_{4}\left(1+H_{1}(t)\right) T
\end{gathered}
$$

for $t \in\left[t_{0}, t^{*}\right]$, where $A_{4} \geq \max \left\{d,(1-\eta) \beta A_{3}\right\}$. Shifting that last term to the other side of the equation yields:

$$
\frac{d T(t)}{d t}+A_{4}\left(1+H_{1}(t)\right) T(t) \geq 0
$$

Since we know

$$
\frac{d}{d t}\left(T(t)+e^{A_{4} \int_{t_{0}}^{t}\left(1+H_{1}(\tau)\right) d \tau}\right) \geq 0,
$$

we find that for $t \in\left[t_{0}, t^{*}\right]$,

$$
T(t) \geq e^{-A_{4} \int_{t_{0}}^{t}\left(1+H_{1}(\tau)\right) d \tau}>0
$$

Therefore $x_{i}(t)>0$, for all $t \in\left[t_{0}, t^{*}\right]$. In particular $x_{i}\left(t^{*}\right)>0$, which is a contradiction and the theorem is proven

Remark 3

1. With this, we have a general idea that the model is sustainable, and can say with certainty that it remains biologically valid as long as it began with biologically-reasonable (i.e, positive) data. This also shows that once infected, it is entirely possible that the virus may continue to exist beneath a detectable threshold without doing any damage.

2. One reason why we choose the strict inequalities for the initial data is that often in biological (or chemical) applications we are interested in the case of solutions where all unknowns are positive. This means intuitively that all elements of the model are 'active'. On the other hand it is sometimes relevant to consider solutions with non-strict inequalities. In fact the statement of the theorem with strict inequalities implies the corresponding statement with non-strict inequalities by using continuous dependence of solutions with respect to initial Data.

\subsection{Existence of Global Solutions}

It will now be shown, with the help of the continuation criterion the existence of global solutions.

Theorem 2.3 The solutions of the Cauchy problem (1)-(4), with positive initial data, exist globally in time in the future that is: on $\left[t_{0},+\infty\right]$.

Proof. To prove this it is enough to show that all variables are bounded on an arbitrary finite interval $\left[t_{0} ; t\right]$. Using the positivity of the solutions is suffices to show that all variables are bounded above.

Taking the sum of equations (1) and (2) shows that:

$$
\frac{d}{d t}(T+I) \leq \lambda
$$

and hence that $T(t)+I(t) \leq T_{0}+I_{0}+\lambda\left(t-t_{0}\right)$. Thus $T$ and $I$ are bounded on any finite interval. The third equation i.e. equation (3), then shows that $V(t)$ cannot grow faster than linearly and is also bounded on any finite interval.

\subsection{Global Boundedness of Solutions}

Theorem 2.4 For any positive solution $(T, I, V)$ of initial value problem (1)-(4) we have:

$$
T(t)+I(t) \leq C_{1} \text { and } V(t) \leq C_{2}
$$

where 
$C_{1}=\max \left\{T_{0}+I_{0}, \frac{\lambda}{\min \{d, \delta\}}\right\}, C_{2}=\max \left\{V_{0}, \frac{(1-\varepsilon) p C_{1}}{c}\right\}$

Proof. According to equations (1) and (2), we have:

$$
\frac{d}{d t}(T+I)=\lambda-d T-\delta I \leq \lambda-\min \{d, \delta\}(T+I)
$$

Gronwall inequality [13] yields:

$$
\begin{gathered}
T+I \leq\left(T\left(t_{0}\right)+I\left(t_{0}\right)\right) e^{-\min \{d, \delta\}\left(t-t_{0}\right)}+\int_{t_{0}}^{t} \lambda e^{-\int_{u}^{t}-\min \{d, \delta\} d r} d u \\
\leq\left(T_{0}+I_{0}\right) e^{-\min \{d, \delta\}\left(t-t_{0}\right)}+\lambda \int_{t_{0}}^{t} e^{-\min \{d, \delta\}(t-u)} d u \\
\leq\left(T_{0}+I_{0}\right) e^{-\min \{d, \delta\}\left(t-t_{0}\right)}+\lambda \frac{e^{-\min \{d, \delta\}(t-t)}-e^{-\min \{d, \delta\}\left(t-t_{0}\right)}}{\min \{d, \delta\}} \\
\leq \max \left\{\left(T_{0}+I_{0}\right), \frac{\lambda}{\min \{d, \delta\}}\right\} e^{-\min \{d, \delta\}\left(t-t_{0}\right)}+\max \left\{\left(T_{0}+I_{0}\right), \frac{\lambda}{\min d, \delta}\right\}\left(1-e^{-\min \{d, \delta\}\left(t-t_{0}\right)}\right) \\
\leq \max \left\{\left(T_{0}+I_{0}\right), \frac{\lambda}{\min \{d, \delta\}}\right\}\left(e^{-\min \{d, \delta\}\left(t-t_{0}\right)}+1-e^{-\min \{d, \delta\}\left(t-t_{0}\right)}\right)
\end{gathered}
$$

therefore $T+I \leq \max \left\{\left(T_{0}+I_{0}\right), \frac{\lambda}{\min \{d, \delta\}}\right\}$.

Another hand, from equation (3), we have:

$$
\begin{gathered}
\frac{d V}{d t}=(1-\varepsilon) p I-c V \leq(1-\varepsilon) p(I+T)-c V \\
\leq(1-\varepsilon) p \max \left\{\left(T_{0}+I_{0}\right), \frac{\lambda}{\min \{d, \delta\}}\right\}-c V \\
\leq(1-\varepsilon) p C_{1}-c V .
\end{gathered}
$$

Once more Gronwall inequality yields:

$$
\begin{gathered}
V(t) \leq V\left(t_{0}\right) e^{-c\left(t-t_{0}\right)}+\int_{t_{0}}^{t}(1-\varepsilon) p C_{1} e^{\int_{u}^{t} c d r} d u \\
\leq V_{0} e^{-c\left(t-t_{0}\right)}+(1-\varepsilon) p C_{1} \int_{t_{0}}^{t} e^{-c(t-u)} d u \\
\leq V_{0} e^{-c\left(t-t_{0}\right)}+(1-\varepsilon) p C_{1} \frac{e^{-c(t-t)}-e^{-c\left(t-t_{0}\right)}}{c} \\
\leq V_{0} e^{-c\left(t-t_{0}\right)}+(1-\varepsilon) p C_{1} \frac{1-e^{-c\left(t-t_{0}\right)}}{c} \\
\leq \max \left\{V_{0}, \frac{(1-\varepsilon) p C_{1}}{c}\right\}\left(e^{-c\left(t-t_{0}\right)}+1-e^{-c\left(t-t_{0}\right)}\right) \\
V(t) \leq \max \left\{V_{0}, \frac{(1-\varepsilon) p C_{1}}{c}\right\}
\end{gathered}
$$

Therefore the theorem is proven.

As consequences of Theorem 2.4 we have:

Remark 4 Let $S$ be a solution of system (1), (2), (3). If $S_{0} \in R \times R_{+}^{3}$ then, the limit of $S(t)$ exits when $t \rightarrow+\infty$. In other words the solution is globally bounded in the future. In particular, $S$ is periodic if and only if $S$ is stationary under the condition that $S(t)$ admits a finite limit when $t$ tends to infinity.

Theorem 2.5 Let $\left(t_{0}, S_{0}=\left(T_{0}, I_{0}, V_{0}\right)\right) \in R \times R_{+}^{3}$ and $\left(\left[t_{0}, T_{1}\right], S=(T, I, V)\right)$ be a maximal solution of the Cauchy problem (1)-(4) $\left(T_{1} \in\left[t_{0},+\infty\right]\right)$. If $T\left(t_{0}\right)+I\left(t_{0}\right) \leq C_{3}$ and $V\left(t_{0}\right) \leq C_{4}$ then the set: 
$\Omega=\left\{(T(t), I(t), V(t)) \in R_{+}^{3}: T(t)+I(t) \leq C_{3}, V(t) \leq C_{4}\right\}$, where $C_{3}=\frac{\lambda}{\min \{d, \delta\}}$ and $C_{4}=\frac{(1-\varepsilon) p \lambda}{c \min \{d, \delta\}}$, is a positively invariant set by system (1), (2), (3).

Proof. Let $t_{1} \in\left[t_{0}, T_{1}\right]$. We shall show that:

1. If $T\left(t_{1}\right)+I\left(t_{1}\right) \leq C_{3}$ then for all $t_{1} \leq t<T_{1}$, $T(t)+I(t) \leq C_{3}$.

2. If $V\left(t_{1}\right) \leq C_{4}$ then for all $t_{1} \leq t<T_{1}, V(t) \leq C_{4}$.

1. Let us suppose that there exists $\varepsilon_{1}>0$ such that: $t_{1} \leq t_{1}+\varepsilon_{1}<T_{1}$,

$$
(T+I)\left(t_{1}+\varepsilon_{1}\right)>C_{3} .
$$

Let $t_{1}^{*}=\inf \left\{t \geq t_{1} /(T+I)(t)>C_{3}\right\}$.

Since $(T+I)\left(t_{1}^{*}\right)=C_{3}$, hence

$(T+I)(t)=C_{3}+\frac{d}{d t}\left(T\left(t_{1}^{*}\right)+I\left(t_{1}^{*}\right)\right)\left(t-t_{1}^{*}\right)+o\left(t-t_{1}^{*}\right)$, $t \rightarrow t_{1}^{*}$. In addition, according to equations (1) and (2) of the system we have:

$$
\frac{d}{d t}(T(t)+I(t))=\lambda-d T-\delta I
$$

which yields:

$$
\begin{gathered}
\frac{d}{d t}(T+I)\left(t_{1}^{*}\right) \leq \lambda-\min \{d, \delta\}(T+I)\left(t_{1}^{*}\right) \\
\leq \lambda-\min \{d, \delta\} C_{3} \\
\leq \lambda-\min \{d, \delta\} \frac{\lambda}{\min \{d, \delta\}} \\
\leq 0
\end{gathered}
$$

hence, there exists $\tilde{\varepsilon}>0$ such that for all $t_{1}^{*} \leq t<t_{1}^{*}+\tilde{\varepsilon}$, $(T+I)(t) \leq C_{3}$, a contradiction. therefore for all $t \in\left[t_{0}, T_{1}\right]$, $(T+I)(t) \leq C_{3}$.

2. Let us suppose that there exists $\varepsilon_{1}>0$ such that: $t_{1} \leq t_{1}+\varepsilon_{1}<T_{1}$,

$$
V\left(t_{1}+\varepsilon_{1}\right)>C_{4}
$$

Let $t_{1}^{*}=\inf \left\{t \geq t_{1} / V(t)>C_{3}\right\}$.

Since $V\left(t_{1}^{*}\right)=C_{3}$, hence

$V(t)=C_{4}+\frac{d V\left(t_{1}^{*}\right)}{d t}\left(t-t_{1}^{*}\right)+o\left(t-t_{1}^{*}\right) \quad, \quad t \rightarrow t_{1}^{*} . \quad$ In addition, according to equation (3), we have:

$$
\frac{d V(t)}{d t}=(1-\varepsilon) p I-c V
$$

which yields:

$$
\begin{gathered}
\frac{d}{d t} V\left(t_{1}^{*}\right) \leq(1-\varepsilon) p(I+T)\left(t_{1}^{*}\right)-c V\left(t_{1}^{*}\right) \\
\leq(1-\varepsilon) p C_{3}-c C_{4} \\
\leq(1-\varepsilon) p \frac{\lambda}{\min \{d, \delta\}}-c \frac{(1-\varepsilon) p \lambda}{c \min \{d, \delta\}} \\
\frac{d}{d t} V\left(t_{1}^{*}\right) \leq 0
\end{gathered}
$$

hence, there exists $\tilde{\varepsilon}>0$ such that for all $t_{1}^{*} \leq t<t_{1}^{*}+\tilde{\varepsilon}$, $V(t) \leq C_{4}$, a contradiction. therefore for all $t \in\left[t_{0}, T_{1}\right]$, $V(t) \leq C_{3}$.

\section{Stability Analyses}

\subsection{Equilibria, Basic Reproduction Number $R_{0}$ and Local Stability}

According to [3], apart from an infection-free equilibrium

$$
E^{0}=\left(T^{0}, 0,0\right) \text { where } T^{0}=\frac{\lambda}{d}
$$

the system (1), (2), (3) has an infected equilibrium during therapy $E^{*}=\left(T^{*}, I^{*}, V^{*}\right)$, where:

$$
\begin{gathered}
T^{*}=\frac{c \delta}{(1-\eta) \beta(1-\varepsilon) p}, I^{*}=\frac{(1-\varepsilon)(1-\eta) \lambda p \beta-d c \delta}{(1-\eta)(1-\varepsilon) p \beta \delta} \\
V^{*}=\frac{(1-\eta)(1-\varepsilon) \lambda p \beta-d c \delta}{(1-\eta) \delta c \beta}=\frac{p(1-\varepsilon)}{c} I^{*}
\end{gathered}
$$

The basic reproduction number $R_{0}$ has been defined in the introduction as the average number of secondary infections that occur when one infective is introduced into a completely susceptible host population $[5,6,14]$. Note that $R_{0}$ is also called the basic reproduction ratio [5] or basic reproductive rate [1]. It is implicitly assumed that the infected outsider is in the host population for the entire infectious period and mixes with the host population in exactly the same way that a population native would mix. Following the method done by [14], we have:

Proposition 3.1 The basic reproduction number $R_{0}$ for model (1) is given by:

$$
R_{0}=(1-\eta)(1-\varepsilon) \frac{\lambda p \beta}{c d \delta}
$$

Now we can express the components of infected equilibrium in term of $R_{0}$. Hence (8) becomes:

$$
T^{*}=\frac{1}{R_{0}} \frac{\lambda}{d}, I^{*}=\frac{c d}{(1-\eta)(1-\varepsilon) p \beta}\left(R_{0}-1\right), V^{*}=\frac{d}{(1-\eta)\left(R_{0}-1\right) \beta}
$$

The following results summarize the main results regarding the local stability of the disease-free steady state $E^{0}$, and the local stability of the infected steady state during therapy $E^{*}$. The proof of these results can be found in [3].

Theorem 3.2 The infection-free steady state $E^{0}$ of model (1), (2), (3) is locally asymptotically stable if $R_{0} \leq 1$ and unstable if $R_{0}>1$.

Theorem 3.3 The infected steady state during the therapy $E^{*}$ of model (1), (2), (3) is locally asymptotically stable if $R_{0}>1$ and unstable if $R_{0}>1$.

\subsection{Global Stability}

In this section, firstly we prove the global stability of the infection-free equilibrium $E^{0}$ of model (1), (2), (3) when the basic reproduction number is less than or equal to unity. And secondly we prove the global stability of infected equilibrium $E^{*}$ whenever it exists. We have seen previously [3] that the unique positive endemic equilibrium exits when the basic 
reproduction number is greater than or equal to unity.

Theorem 3.4

1. The infection-free steady state $E^{0}$ of model (1), (2), (3) is globally asymptotically stable if the basic reproduction number $R_{0} \leq 1$ and unstable if $R_{0}>1$.

2. The infected steady state during therapy $E^{*}$ of model (1), (2), (3) is globally asymptotically stable if the basic reproduction number $R_{0} \leq 1$.

Proof.

1. Consider the Lyapunov function:

$$
L_{1}(T, I, V)=T-T^{0}-T^{0} \ln \frac{T}{T^{0}}+I+\frac{\delta}{(1-\varepsilon) p} V .
$$

$L_{1}$ is defined, continuous and positive definite for all $T>0, I>0, V>0$. Also, the global minimum $L_{1}=0$ occurs at the infection free equilibrium $E^{0}$. Further, function $L_{1}$, along the solutions of system (1), (2), (3) satisfies:

$\frac{d L_{1}}{d t}=\lambda-d T-\frac{T^{0}}{T} \lambda+d T^{0}+(1-\eta) \beta V T^{0}-\frac{c \delta}{(1-\varepsilon) p} V$.

Further collecting terms, we have

$$
\begin{gathered}
\frac{d L_{1}}{d t}=\lambda\left(2-\frac{T}{T^{0}}-\frac{T^{0}}{T}\right)+\left(R_{0}-1\right) \frac{c \delta}{(1-\varepsilon) p} V . \\
L_{2}(T, I, V)=T-T^{*}-T^{*} \ln \frac{T}{T^{*}}+I-I^{*}-I^{*} \ln \frac{I}{I^{*}}+\frac{\delta}{(1-\varepsilon) p}\left(V-V^{*}-V^{*} \ln \frac{V}{V^{*}}\right) .
\end{gathered}
$$

The time derivative of $L_{2}$ along the trajectories of system (1), (2), (3) is:

$$
\frac{d L_{2}}{d t}=\frac{d T}{d t}-\frac{T^{*}}{T} \frac{d T}{d t}+\frac{d I}{d t}-\frac{I^{*}}{I} \frac{d I}{d t}+\frac{\delta}{(1-\varepsilon) p} \frac{d V}{d t}-\frac{\delta}{(1-\varepsilon) p} \frac{V^{*}}{V} \frac{d V}{d t}
$$

Collecting terms, and canceling identical terms with opposite signs, yields

$$
\begin{gathered}
\frac{d L_{2}}{d t}=\lambda-d T-\lambda \frac{T^{*}}{T}+d T^{*}+(1-\eta) \beta V T^{*}-(1-\eta) \beta V T \frac{I^{*}}{I}+\delta I^{*}-\frac{c V \delta}{(1-\varepsilon) p}-\delta \frac{V^{*}}{V} I+\frac{c \delta}{(1-\varepsilon) p} V^{*} \\
=d R_{0} T^{*}-d T^{*} \frac{T}{T^{*}}-d R_{0} T^{*} \frac{T^{*}}{T}+d T^{*}+(1-\eta) \beta V^{*} T^{*} \frac{V}{V^{*}}+\delta I^{*}-(1-\eta) \beta V^{*} T^{*} \frac{T}{T^{*}} \frac{V}{V^{*}} \frac{I^{*}}{I}-\frac{c \delta}{(1-\varepsilon) p} \frac{V}{V^{*}} V^{*}-\delta \frac{V^{*}}{V} \frac{I}{I^{*}} I^{*}+\frac{c \delta}{(1-\varepsilon) p} V^{*}, \\
\quad \operatorname{according} \operatorname{to}(9), \\
=d T^{*}\left(R_{0}-\frac{T}{T^{*}}-R_{0} \frac{T^{*}}{T}+1\right)+(1-\eta) \beta V^{*} T^{*}\left(\frac{V}{V^{*}}-\frac{V T I^{*}}{V^{*} T^{*} I}\right)+\delta I^{*}\left(2+\frac{V}{V^{*}}-\frac{V^{*} I}{V I^{*}}\right) \\
=d T^{*}\left(R_{0}-\frac{T}{T^{*}}-R_{0} \frac{T^{*}}{T}+1\right)+\delta I^{*}\left(\frac{V}{V^{*}}-\frac{V T I^{*}}{V^{*} T^{*} I}\right)+\delta I^{*}\left(2+\frac{V}{V^{*}}-\frac{V^{*} I}{V I^{*}}\right), \\
\frac{d L_{2}}{d t}=d T^{*}\left(R_{0}-\frac{T}{T^{*}}-R_{0} \frac{T^{*}}{T}+1\right)+\delta I^{*}\left(2-\frac{V^{*} I}{V I^{*}}-\frac{V T I^{*}}{V^{*} T^{*} I}\right)+\delta I^{*}\left(1-\frac{T^{*}}{T}\right)-\delta I^{*}\left(1-\frac{T^{*}}{T}\right) \\
=d T^{*}\left(R_{0}-\frac{T}{T^{*}}-R_{0} \frac{T^{*}}{T}+1\right)+\delta I^{*}\left(3-\frac{T^{*}}{T}-\frac{V^{*} I}{V I^{*}}-\frac{V T I^{*}}{V^{*} T^{*} I}\right)-d T^{*}\left(R_{0}-1\right)\left(1-\frac{T^{*}}{T}\right), \\
\operatorname{since} \delta I^{*}=d T^{*}\left(R_{0}-1\right), \\
\frac{d L_{2}}{d t}=d T^{*}\left(2-\frac{T}{T^{*}}-\frac{T^{*}}{T}\right)+\delta I^{*}\left(3-\frac{T^{*}}{T}-\frac{V^{*} I}{V I^{*}}-\frac{V T I^{*}}{V^{*} T^{*} I}\right) \cdot \leq 0 .
\end{gathered}
$$


Since the terms between the brackets are less than or equal to zero by the inequality (the geometric mean is less than or equal to the arithmetic mean)

$$
\sqrt[3]{a_{1} a_{2} a_{3}}-\left(a_{1}+a_{2}+a_{3}\right) \leq 0, a_{i} \geq 0, i=1,2,3 .
$$

It should be noted that $\frac{d L_{2}}{d t}=0$ holds if and only if $(T, I, V)$ take the steady states values $\left(T^{*}, X^{*}, V^{*}\right)$ Therefore, according to Lasalle invariance principle, the infected equilibrium $E^{*}$ is globally asymptotically stable.

\section{Numerical Simulation}

Some numerical simulations have been done in the case $R_{0}<1$ to confirm theoretical result obtain on global stability for the uninfected equilibrium.

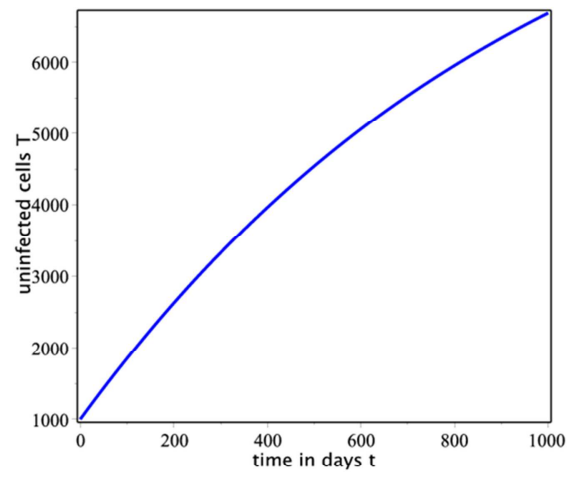

Numerical solution curve for the uninfected cells

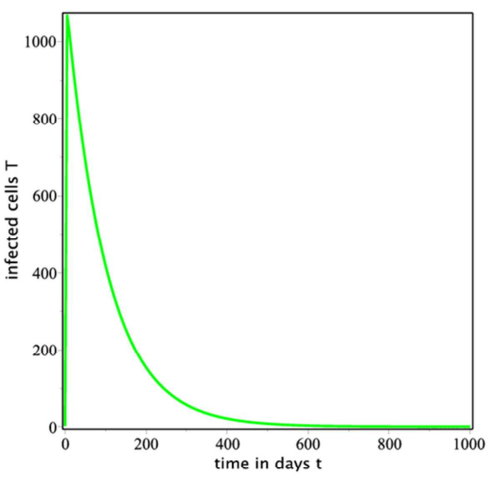

Numerical solution curve for the infected cells

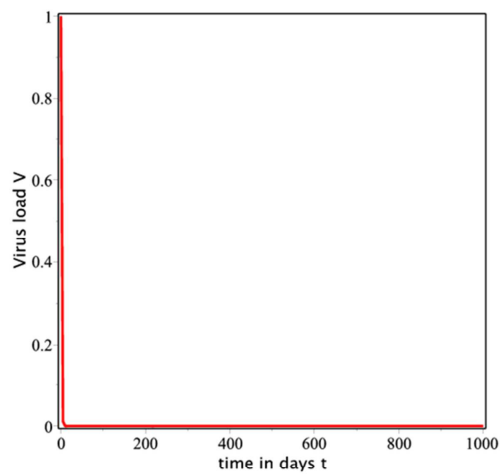

Numerical solution curve for the virus load

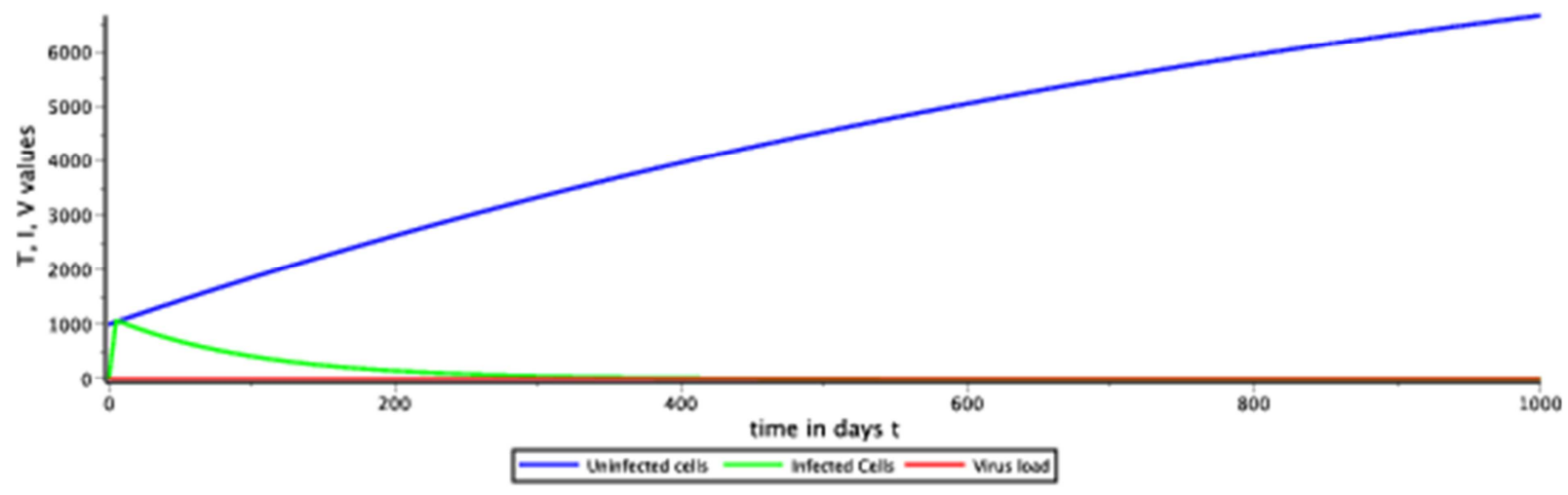

Figure 2. Numerical simulation of the HCV model in 1000 days.

We run simulations using the initial conditions: $T=10^{3}$, $I=2$ and $V=1$ and the following parameter values: $\lambda=10 ; \eta=\frac{1}{10000000} ; \beta=2.4 \times 10^{-8} ; \delta=0.01 ; d=$ $0.001 ; \varepsilon=0.00000001 ; c=0.9, p=0.000000001$.

\section{Conclusion}

In this paper, we have extended the first part of the work done by Chong et al. in [3] where they only studied the local stability of the fundamental mathematical model of hepatitis $\mathrm{C}$ virus infection with treatment. We constructed suitable Lyapunov functions to prove that if the reproduction number $R_{0} \leq 1$ the HCV- uninfected equilibrium point is globally asymptocally stable; and if $R_{0}>1$ the HCV-infected equilibrium point is globally asymptotically stable. Finally we performed numerical simulations to illustrate theoretical results obtained at $\mathrm{HCV}$-uninfected equilibrium point. It would be interesting to incorporate time delay, diffusion phenomenon or random phenomenon into the cyrrent model. Also, using the standard incidence function instead of mass action principle could be a serious issue. These two challenges will be the concerns of future investigation.

\section{Acknowledgements}

I am grateful to Professor Alan Rendall for valuable and tremendous discussions. I wish to thank him for introducing me to Mathematical Biology and to its relationship with Mathematical Analysis.

\section{References}

[1] R. M. Anderson and R. M. May, eds. 1991. Infectious Diseases of Humans: Dynamics and Control, Oxford University Press, Oxford, UK.

[2] Chatterjee, A., Guedj, J., \& Perelson, A. S. (2012). Mathematical modelling of $\mathrm{HCV}$ infection: what can it teach us in the era of direct-acting antiviral agents? Antiviral Therapy, 17 (6PtB), 1171-1182. http://dx.doi.org/10.3851/IMP2428. 
[3] Chong, Maureen Siew Fang, Shahrill, Masitah, Crossley, Laurie and Madzvamuse, Anotida, 2015 The stability analyses of the mathematical models of hepatitis $C$ virus infection. Modern Applied Science, 9 (3). pp. 250-271. ISSN 1913-1844.

[4] Dahari, H., Lo, A., Ribeiro, R. M., \& Perelson, A. S. (2007). Modeling hepatitis $C$ virus dynamics: Liver regeneration and critical drug efficacy. Journal of Theoretical Biology, 247, 371-381. http://dx.doi.org/10.1016/j.jtbi.2007.03.006.

[5] O. Diekmann, J. A. P. Heesterbeek, and J. A. J. Metz, 1990. On the definition and the computation of the basic reproduction ratio $R 0$ in models for infectious diseases in heterogeneous populations, J. Math. Biol., 28, pp. 365-382.

[6] K. Dietz, 1988. Density dependence in parasite transmission dynamics, Parasit. Today, 4, pp. 91-97.

[7] Guedj, J., \& Neumann, A. U. (2010). Understanding hepatitis C viral dynamics with direct-acting antiviral agents due to the interplay between intracellular replication and cellular infection dynamics. Journal of Theoretical Biology, 267 (3), 330-340. http://dx.doi.org/10.1016/j.jtbi.2010.08.036.

[8] Khalil, H., 2002. Nonlinear Systems, 3rd edn. Prentice Hall, New York.

[9] Neumann, A. U., Lam, N. P., Dahari, H., Gretch, D. R., Wiley, T. E., Layden, T. J., \& Perelson, A. S. (1998). Hepatitis C viral dynamics in vivo and the antiviral efficacy of interferon- therapy. $\quad$ Science, $\quad 282, \quad 103-107$. http://dx.doi.org/10.1126/science.282.5386.103.

[10] Reluga, T. C., Dahari, H., \& Perelson, A. S. (2009). Analysis of hepatitis $C$ virus infection models with hepatocyte homeostasis. SIAM Journal on Applied Mathematics, 69 (4), 999-1023. http://dx.doi.org/10.1137/080714579.

[11] Rong, L., \& Perelson, A. S. (2013). Mathematical analysis of multiscale models for hepatitis $\mathrm{C}$ virus dynamics under therapy with direct-acting antiviral agents. Mathematical Biosciences, 245 (1), 22-30. http://dx.doi.org/10.1016/j.mbs.2013.04.012.

[12] L. B. Seef, 2002. Natural history of chronic hepatitis $C$, Hepatology, 36: S35-S46.

[13] Sever Silvestru Dragomir (2013).. Some Gronwall Type Inequalities and Applications, NOVA, Melbourne.

[14] P. van den Driessche and James Watmough, 2002. Reproduction numbers and sub-threshold endemic equilibria for compartmental models of disease transmission. Mathematical Biosciences 180 29-48.

[15] WHO, Guidelines for the sreening, care and treatement of persons with hepatitis C infection, ISBN 978924154875 5, NLM classification: WC 536, 2014. 